Article

\title{
Breath Sensor Technology for the Use in Mechanical Lung Ventilation Equipment for Monitoring Critically Ill Patients
}

\author{
Manohar Prasad Bhandari ${ }^{1, *}$, Viktors Veliks ${ }^{1}$, Ilmārs Stonāns ${ }^{1}$, Marta Padilla ${ }^{2}$, Olegs Šuba ${ }^{3}$, Agija Svare ${ }^{3}$, \\ Inga Krupnova ${ }^{3}$, Nikita Ivanovs ${ }^{3}$, Dina Bēma ${ }^{1}$, Jan Mitrovics ${ }^{2}$ and Mārcis Leja ${ }^{1,3,4, *(\mathbb{C})}$ \\ 1 Institute of Clinical and Preventive Medicine, University of Latvia, LV-1586 Riga, Latvia; \\ viktors.veliks@lu.lv (V.V.); ilmars.stonans@lu.lv (I.S.); dina.bema@lu.lv (D.B.) \\ 2 JLM Innovation GmbH, D-72070 Tubingen, Germany; marta.padilla@jlm-innovation.de (M.P.); \\ jan.mitrovics@jlm-innovation.de (J.M.) \\ 3 Riga East University Hospital, LV-1038 Riga, Latvia; olegs.suba@aslimnica.lv (O.Š.); agija.r@gmail.com (A.S.); \\ inga.krupnova@aslimnica.lv (I.K.); nikita.ivanovs@gmail.com (N.I.) \\ 4 Faculty of Medicine, University of Latvia, LV-1586 Riga, Latvia \\ * Correspondence: manohar_prasad.bhandari@lu.lv (M.P.B.); marcis.leja@lu.lv (M.L.)
}

\section{check for}

updates

Citation: Bhandari, M.P.; Veliks, V.; Stonāns, I.; Padilla, M.; Šuba, O.; Svare, A.; Krupnova, I.; Ivanovs, N, Bēma, D.; Mitrovics, J.; et al. Breath Sensor Technology for the Use in Mechanical Lung Ventilation

Equipment for Monitoring Critically Ill Patients. Diagnostics 2022, 12, 430 https://doi.org/10.3390/ diagnostics 12020430

Academic Editor: Chao-Min Cheng

Received: 30 December 2021

Accepted: 6 February 2022

Published: 7 February 2022

Publisher's Note: MDPI stays neutral with regard to jurisdictional claims in published maps and institutional affiliations.

Copyright: (C) 2022 by the authors. Licensee MDPI, Basel, Switzerland. This article is an open access article distributed under the terms and conditions of the Creative Commons Attribution (CC BY) license (https:// creativecommons.org/licenses/by/ $4.0 /$ )

\begin{abstract}
Background: The need for mechanical lung ventilation is common in critically ill patients, either with COVID-19 infection or due to other causes. Monitoring of patients being ventilated is essential for timely and improved management. We here propose the use of a novel breath volatile organic compound sensor technology to be used in a mechanical lung ventilation machine for this purpose; the technology was evaluated in critically ill COVID-19 patients on mechanical lung ventilation. Methods: Based on the consistency results of our study data, the breath sensor device with metal oxide gas sensors and environment-controlling sensors was mounted on the ventilation exhaust port of the ventilation machine; this allowed to ensure additional safety since the device was placed outside the contour between the patient and equipment. Results: The sensors allowed stable registration of the signals for up to several weeks for 10 patients in total, depending on the storage amount; a proportion of patients were intubated or received tracheostoma during the evaluation period. Future studies are on the way to correlate sensor readings to other parameters characterizing the severity of the patient condition and outcome. Conclusions: We suppose that such technology will allow patient monitoring in real-time for timely identification of deterioration, potentially requiring some change of management. The obtained results are preliminary and further studies are needed to examine their clinical significance.
\end{abstract}

Keywords: VOC sensors; mechanical ventilation; exhaled breath; COVID-19; patient monitoring

\section{Introduction}

Mechanical ventilation is a life-saving medical intervention in patients experiencing respiratory failure in the case of critical illness, including but not limited to COVID-19. These patients are at risk of multiple complications, and require intensive monitoring to identify signs of clinical worsening and to minimize the risk of iatrogenic harm [1]. Pulse oximetry, capnography, monitoring of driving pressure, transpulmonary pressure, and the pressure-volume loop, as well as adjustment of airway cuff pressures are being used in these patients; however, each of the methods has its limitations [1]. It is important to ensure reliable monitoring of critically ill patients, including those with COVID-19, during mechanical ventilation to minimize harm to the patients.

The rapid detection of COVID-19-specific breath volatile organic compounds (VOCs) would be a great step forward for diagnostic purposes and for monitoring disease progression or responses to conventional or investigational drugs. Here, we present a novel sensor-based VOC technology to be assembled within the artificial ventilation equipment for severely ill patients, including those with COVID-19. The major advantages of the VOCs 
sensor device combined with the mechanical ventilation system are its simple and robust design, non-invasiveness, easy operation, fast response, reusability, application in hospital usage, convenient data acquisition and data exchange, and low manufacturing costs.

The rapid spread and transmission of COVID-19 has threatened global health, leading to an urgent need of new technology for its monitoring and diagnosis. The analysis of VOCs in exhaled human breath has become a growing field of research in recent years because of the advances in analytical techniques and nanotechnology [2,3]. These compounds may provide valuable information about the metabolic activity of the enzymes or pathological processes of any disease. The VOCs profile associated with COVID-19 infection will provide information on potential prognostic features. Metal oxide (MOX) gas sensors are most frequently used in VOC testing approaches. They are usually cross-reactive and have high sensitivity to VOCs. Moreover, such sensors are sensitive under high humidity conditions, which is an important requirement for exhaled breath analysis. MOX-based chemiresistive sensors have been receiving attention due to their high potential for miniaturization of portable diagnostic tools. Sensor technology application for VOCs detection in human breath for disease monitoring is easy-to-use, non-invasive, fast, and low-cost [4-6]. An array of semiconducting MOX sensors along with methods of pattern recognition and analytics is capable of detecting a compendium of VOCs in exhaled breath. Each sensor responds to a range of VOCs, which allows the sensing and analysis of their pattern from a mixture of compounds. The isolation of disease-specific VOCs and their detection via breathomics and novel sensing materials highlights the clinical applicability of exhaled breath monitoring $[7,8]$.

Few previous studies have addressed the use of VOCs in the monitoring of ventilated patients, suggesting this to be a valuable strategy for non-invasive control and optimization of ventilation strategies [9-11]. This has been further endorsed in studies involving COVID19 patients by suggesting that particular breath chemistries (namely, methylpent-2-enal, 2,4-octadiene 1-chloroheptane, and nonanal) could be identified in the breath of these patients developing acute respiratory distress syndrome (ARDS), a condition that can be treated with mechanical ventilation [12]. Importantly, mechanical lung ventilation is associated with a significantly increased risk of hospital infection [13], which is also the case for COVID-19 patients [14]. A study involving the use of a novel breath detector device with a catalytically active, resistive chemosensor that is highly selective to $\mathrm{NO}$ and ammonia was able to detect the distinct breath signature of COVID-19 within 15 seconds [15]. A multiplexed nanomaterial-based hybrid sensor array utilizing advanced machine learning and feature extraction algorithms enabled the detection and monitoring of COVID-19 from exhaled breath [16]. This gave us a good reason to hypothesize that sensor-based VOC breath technology could become a reliable tool for monitoring critically ill patients, including those with COVID-19.

However, multiple challenges with the application of this technology for the abovementioned purposes do exist. Sensor readings could be substantially influenced by breath humidity [17], medications, clinical parameters, inter/intraperson variability of the VOC profile, and other external variables, such as patient condition, coughing, mucous, ventilation tube movements, and mechanical issues in the lung ventilation machine [18]. In addition, cross-sensitivities to environmental and external variables influencing the environmental sensors affect the gas sensors response, as well as sensor drift and sensor warmingup $[19,20]$. To counteract such unwanted effects, appropriate signal pre-processing can be applied and a multivariate predictive model is built using statistical and machine learning methods. These limitations are addressed by the proposed breath sensor technology, so that the effect of the evolution on the patients' state and breath characteristics can be isolated and thus monitored.

\section{Materials and Methods}

We designed a sensor-based VOC technology and performed the initial validation of the technology in critically ill patients with COVID-19 infections being managed in one of 
the two departments of Riga East Clinical University Hospital-Clinic of Toxicology and Sepsis or Latvia Infectology Centre. The details of its design, the placement of the sensor device in the mechanical lung ventilation machine where it can be best used, sensor set, requirements that are met by this modular device in the clinical setup, data acquisition, and sensor reading results are explained.

\subsection{Design and Engineering of the Sensor Device}

The design of the technology was performed by JLM Innovation GmbH (Tübingen, Germany) based on the definition of the need defined by the clinical partners. The sensor device comprises the environmental sensors that measure temperature, pressure and humidity, and a set of MOX gas sensors with different specificities measuring VOC patterns present in the patient's breath. The registration device is built on a Raspberry Pi platform and the data recording is achieved through a Python script.

The gas sensors are housed in a Clear-Guard MIDI disposable filter housing with a Luer port that is connected to the mechanical lung ventilation machine. The filter with the sensors is disposable, while the sensor signal registration device, which is not in direct contact with the patient's breath, is reusable. Figure 1A shows a model of a Clear-Guard MIDI breathing HME (Heat and Moisture Exchanger) filter (Intersurgical Ltd., Wokingham, $\mathrm{UK})$, wherein the sensor device is integrated. A complete set-up of the sensor device adapted for use in the mechanical ventilation equipment is shown in Figure 1B. Figure 1C shows the sensor board connected to a micro-USB cable for the sensor signal recording. The device can be controlled and data acquisition could be done via the JLMlogSP software using a Wi-Fi transmitter. This would make the sensor device more efficient to be deployed at the hospital.

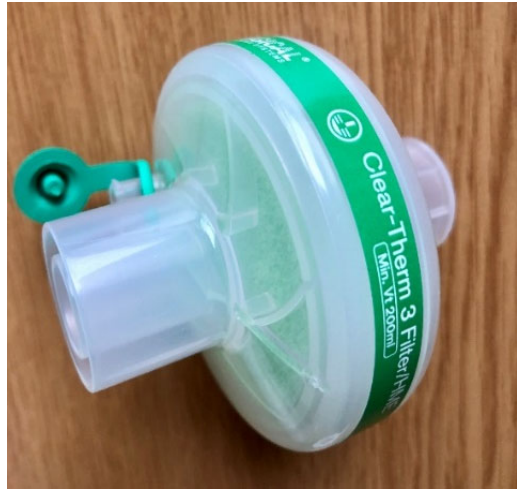

(A)

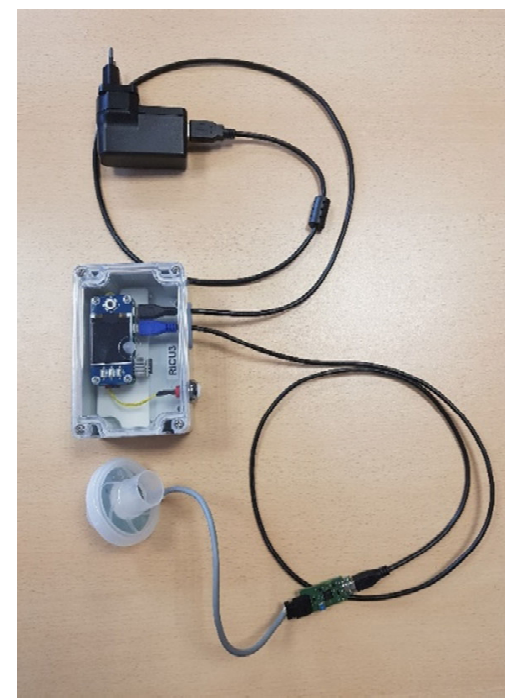

(B)

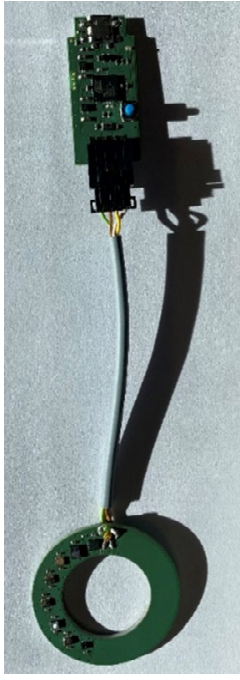

(C)

Figure 1. (A) Clear-Guard MIDI breathing filter with Luer port; (B) sensor device adapted for use in the mechanical lung ventilation machine; the device embedded in the Clear-Guard MIDI breathing filter, a sensor signal registration device (Raspberry Pi), and a power adapter; (C) sensor board connected to a micro-USB cable.

The technical design of the sensor device electronics is shown in Figure 2. All of the sensors are embedded on the right side of the device. 


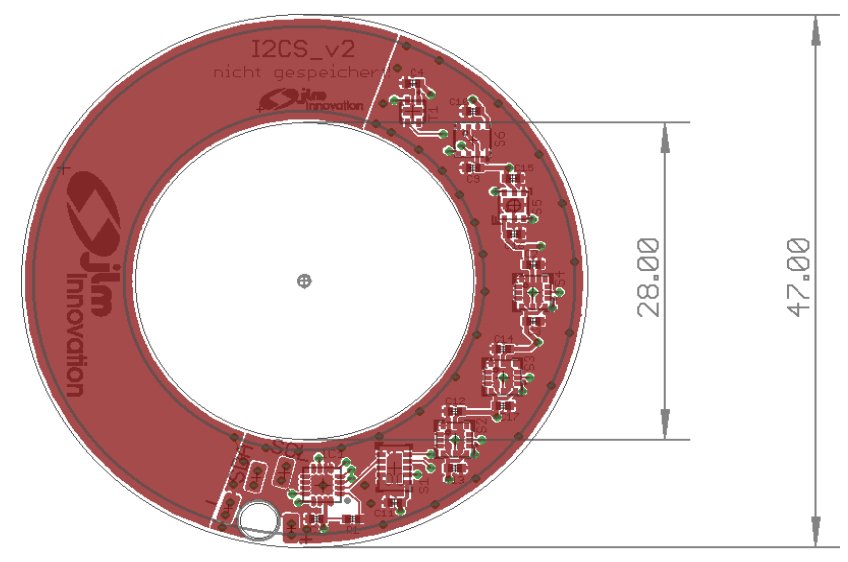

Figure 2. Technical design of the sensor device (all dimensions are in $\mathrm{mm}$ ). The sensors are on the right side.

The sophisticated and unique use-case of breath VOC sensor technology having incorporated into the mechanical lung ventilation machine and meeting various specifications was tested and evaluated in real conditions on patients suffering from COVID-19.

\subsection{Placement of the Sensor Device}

Our team of medical doctors, researchers, and engineers explored the concerns around the appropriate placement of the sensor device in the mechanical lung ventilation machine (HAMILTON-C6, Hamilton Medical AG, Bonaduz, Switzerland) to address the efficacy of the device. For this purpose, possible allocations of the sensor device in the ventilation machine and the stability of the MOX gas sensor readings at those locations were evaluated (Figure 3). An array of semiconducting MOX gas sensors and an environmental sensor module was integrated into the ventilation machine to help monitor patients' state and recovery.

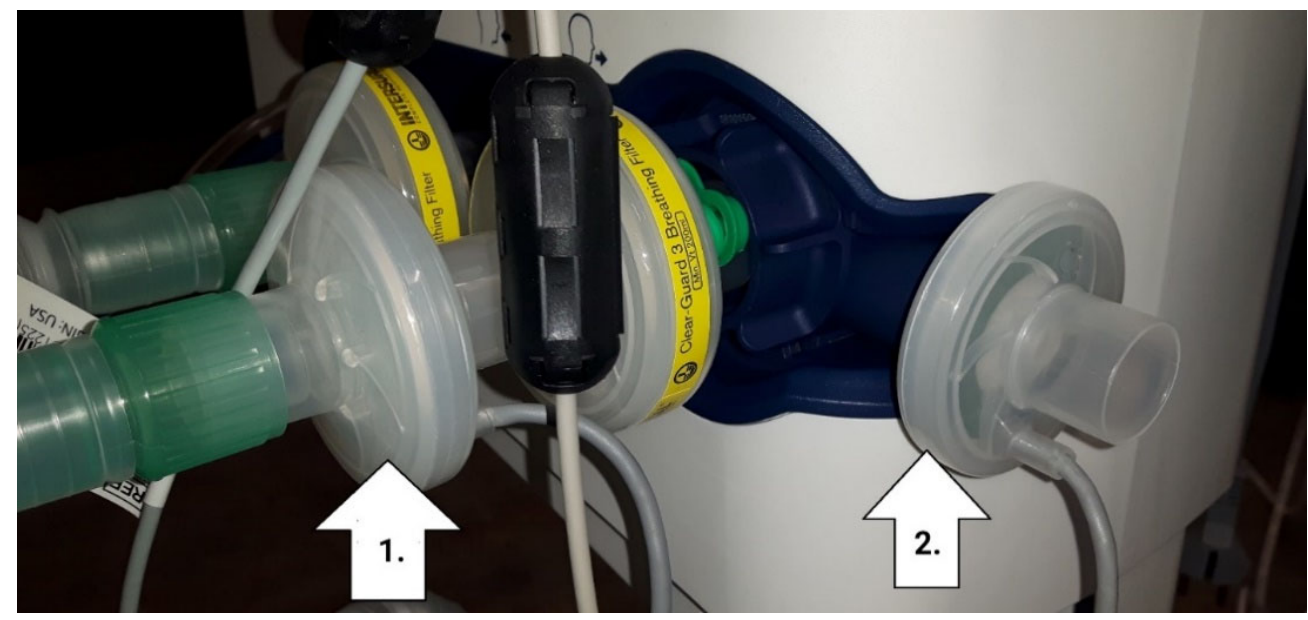

Figure 3. Sensor device placement at the outlet of the mechanical lung ventilation machine (position 2) in a real operating condition in a hospital setting, which was adopted. Another potential location (position 1) of the breath contour before the ventilation machine was also evaluated.

Taking into consideration the potential risk of contamination, safety, practicality, breath humidity, and sensor interaction with the infectious material, the outflow part of the ventilation machine was identified and selected to be the optimal place for sensor localization (on the right side of Figure 3, position 2). The installation of the sensor module on the ventilation exhaust port or outlet was carried out and the breathing measurement data were obtained, hence ensuring the normal operation of the mechanical ventilator in 
safety conditions for the patients and the clinicians. Since the sensor readings were stable, the outlet of the ventilation machine was chosen to exclude any contact to the airflow between the patient and the ventilator. The humidity was also measured, and it did not have any impact on the chosen position. There, the sensor device does not come into contact with the patient, does not interfere with the treatment, and is less exposed to the adverse conditions.

Figure 4 shows a schematic representation of the whole system including the ventilation airways and modules, sensor placement, sensors signal recordings, and data exchange using a Wi-Fi transmitter. The construction and localization of the breath sensor device is designed to enable the use of the instrumentation for monitoring severely ill patients connected to the mechanical lung ventilation machine. From the patient, the expiration air flows through the intubation tubes and relevant bacterial filters into the VOC sensor block located at the exhaust port of the mechanical lung ventilation machine. The airflow from the sensor system does not return to the patient. The breathing measurement data from the sensor readings are recorded using a data cable and a Wi-Fi transmitter for further processing and analysis. The Wi-Fi transmitter works only while performing the data exchange and it is turned off during the observation. This is important for the safety of other medical devices. The sensor device can be connected to a Windows PC and controlled via the in-house JLMlogSP software. The output data are generated in a text file format, which can be processed using multivariate signal processing for further analysis and visualizations.

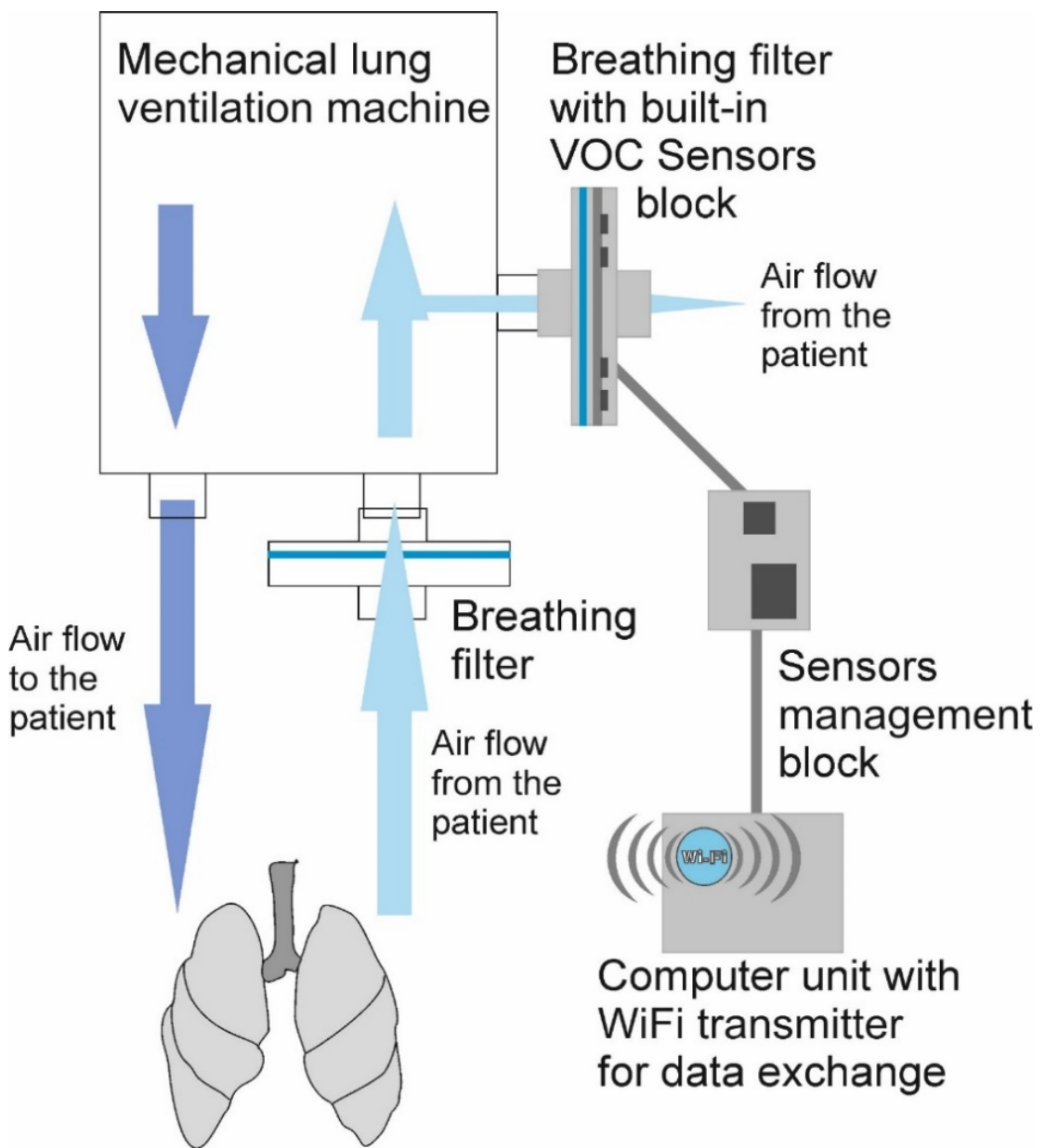

Figure 4. Schematic of the VOC sensor device placement including the ventilation airways and sensors' signal acquisition for the monitoring of COVID-19 patients. 


\subsection{Sensors Characteristics}

The sensor device is equipped with environmental sensors that measure temperature, pressure, and relative humidity, and an array of chemiresistive MOX gas sensors. Table 1 summarizes the description of the sensors used in the breath sensor module together with their main features. The gas sensor array contains six digital gas sensors: one sensor with two outputs for VOC sensing (SGP30), two sensors with one output for VOC sensing (CCS811 and BME680), and three sensors each modulated at three temperature levels through heater voltage regulation (IDT-ZMOD). Modulation in temperature is an interesting property of the MOX sensors, which all need to be heated so that the chemical reactions can take place at their sensing layer. MOX sensors show different sensitivities when heated at different temperatures. For this reason, having three sensors (IDT-ZMOD) heated at three different temperatures is equivalent to having nine sensors. Therefore, the array gives 13 sensor signals which provide a specific fingerprint on the VOC content in the exhaled human breath. All MOX gas sensors have broad sensitivity to the VOCs in the breath, responding to the presence of many types of VOCs produced by internal biochemical processes of the human metabolism [21].

Table 1. Description of the sensors used in the sensor device.

\begin{tabular}{|c|c|c|c|c|}
\hline Manufacturer & Sensor Type/Model & Technology & Number/Subtype & Sensor Main Feature \\
\hline Renesas/IDT & ZMOD4410A & MOX & 3 & $\begin{array}{l}\text { One output for VOCs } \\
\text { at three different temperatures }\end{array}$ \\
\hline Renesas/IDT & ZMOD4410C & MOX & 3 & $\begin{array}{l}\text { One output for VOCs } \\
\text { at three different temperatures }\end{array}$ \\
\hline Renesas/IDT & ZMOD4510B & MOX & 3 & $\begin{array}{l}\text { One output for VOCs } \\
\text { at three different temperatures }\end{array}$ \\
\hline AMS/ScioSense & CCS811 & MOX & 1 & One output for VOCs \\
\hline Sensirion & SGP30 & MOX & 2 & $\begin{array}{l}\text { Two outputs for EtOh and for } \mathrm{H}_{2} \\
\text { One output for VOCs, three }\end{array}$ \\
\hline $\mathrm{BOSCH}$ & BME680 & MOX & 1 & $\begin{array}{l}\text { outputs for environmental } \\
\text { variables: temperature, pressure, } \\
\text { and humidity }\end{array}$ \\
\hline AMS/ScioSense & ENS210 & - & 2 & $\begin{array}{c}\text { Two outputs for temperature } \\
\text { and humidity }\end{array}$ \\
\hline
\end{tabular}

These sets of sensors were selected for the designated task based on sensing characteristics, low power consumption, technological quality, low cost, and compatibility with medical applications. The sensor module was connected to the exhaust port of the mechanical lung ventilation machine and allowed continuous measurements of exhaled breath and acquisition of signals every second during the non-invasive ventilation of COVID-19 patients.

\section{Results}

\subsection{Sensor Signal Recording}

After the approval of the sensor device placement in the mechanical lung ventilation machine, sensor placement in a hospital setting was started. The device was tested on patients with severe COVID-19 infection under treatment. The data were collected by small Raspberry Pi, which later transmitted the data via a Wi-Fi transmitter. The breathing measurement data from the patients were recorded which could be used to monitor changes in the VOCs present in the exhaled breath of the patients connected to the ventilation machine. The presence of endogenous VOCs in exhaled breath gives knowledge about their pathophysiological origins and the biochemical pathways involved in disease development. They serve as preclinical biomarkers of various diseases [22].

Figure 5 reports an example of signals acquired from all the sensors during the ventilation of a COVID-19 patient in a real operating condition for one week, with additional 
external measurement information on the ratio of peripheral arterial oxygen saturation to the fraction of inspired oxygen $\left(\mathrm{SpO}_{2} / \mathrm{FiO}_{2}\right)$, the ratio of partial pressure arterial oxygen to the fraction of inspired oxygen $\left(\mathrm{PaO}_{2} / \mathrm{FiO}_{2}\right)$, the pulse, and the mean arterial pressure (MAP) data. The acquired raw sensor signals in resistance were normalized to compare the sensor responses. It can be observed that all the gas sensors, each with its own response time, showed a good correlation in different conditions of the patient, with the ability to catch every single respiratory act. All sensors were stable during the long-time measurement period and delivered good resolution readings; even before the sensors became stable, the sensor response signals could be identified. By comparing the temperature, humidity, and pressure sensor data with the gas sensor data, it can be seen that the gas sensors pick up small independent signals.
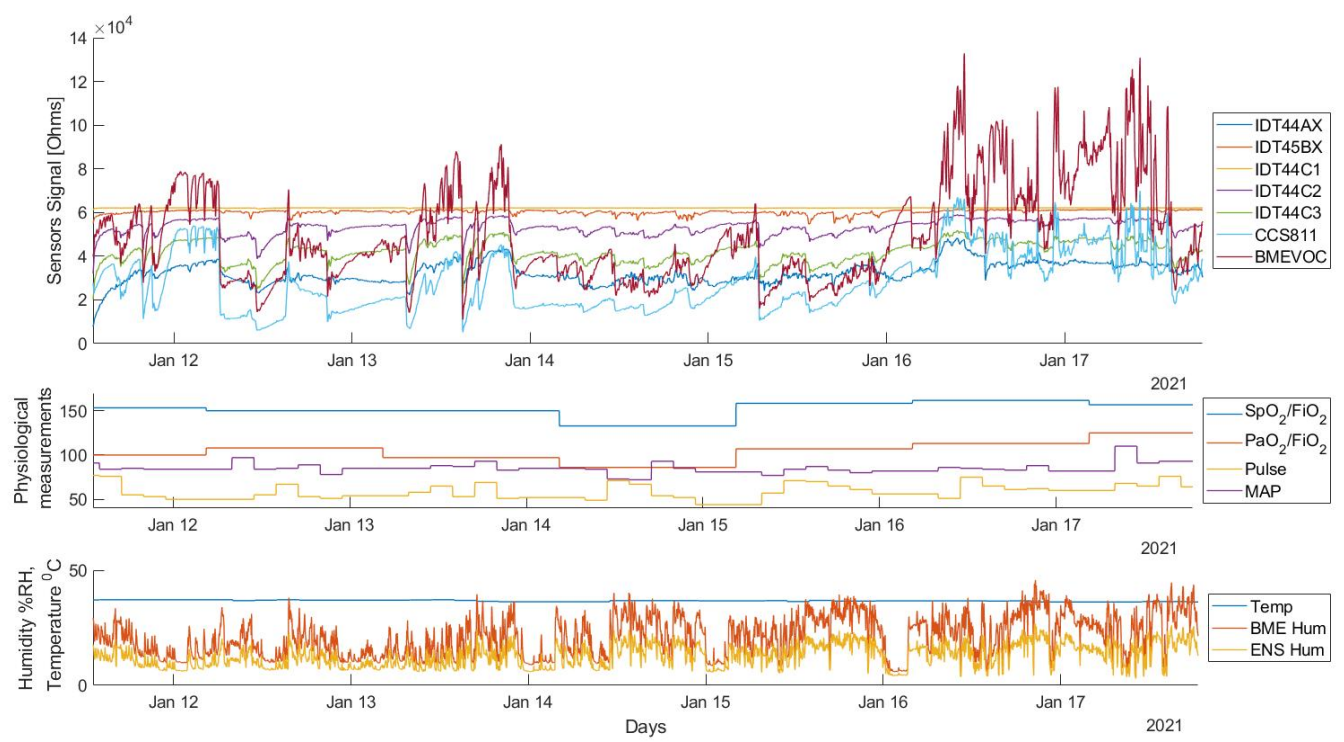

Figure 5. Sensor signals obtained during the ventilation of a patient (total recording time or full registration period). The measurements refer to one-week period.

The signals from the MOX gas sensors vary during the ventilation period to exhaled VOC content indicating a promising sensitivity to exhaled VOCs. Multivariate signal processing of the sensors array data will help to predict the condition/course of the disease, correlating the obtained sensor data with the clinical information, which will help the doctors adjust their treatment, for example by adding medicines with potential antiviral activity. At the same time, the sensor measurement data could help diagnose the potential nosocomial infections and antibacterial management in a timely manner. It is necessary to filter out the indicators responding to the change of the medical intervention of the patient that affects the sensors' response.

Figure 6 shows a segment of the recorded sensors' signals towards environmental sensor data for one patient during $24 \mathrm{~h}$ of observation. Environmental sensors record temperature and humidity changes in the breath of the patient and are measured by two sensors: the first one is an ENS210 located separately on the board, and the second is a part of BME680 sensors. The temperature sensor in the BME680 is affected by the sensor heating, and for this reason, an external sensor ENS210 is included in the device. Both humidity sensors show similar patterns of humidity changes. 


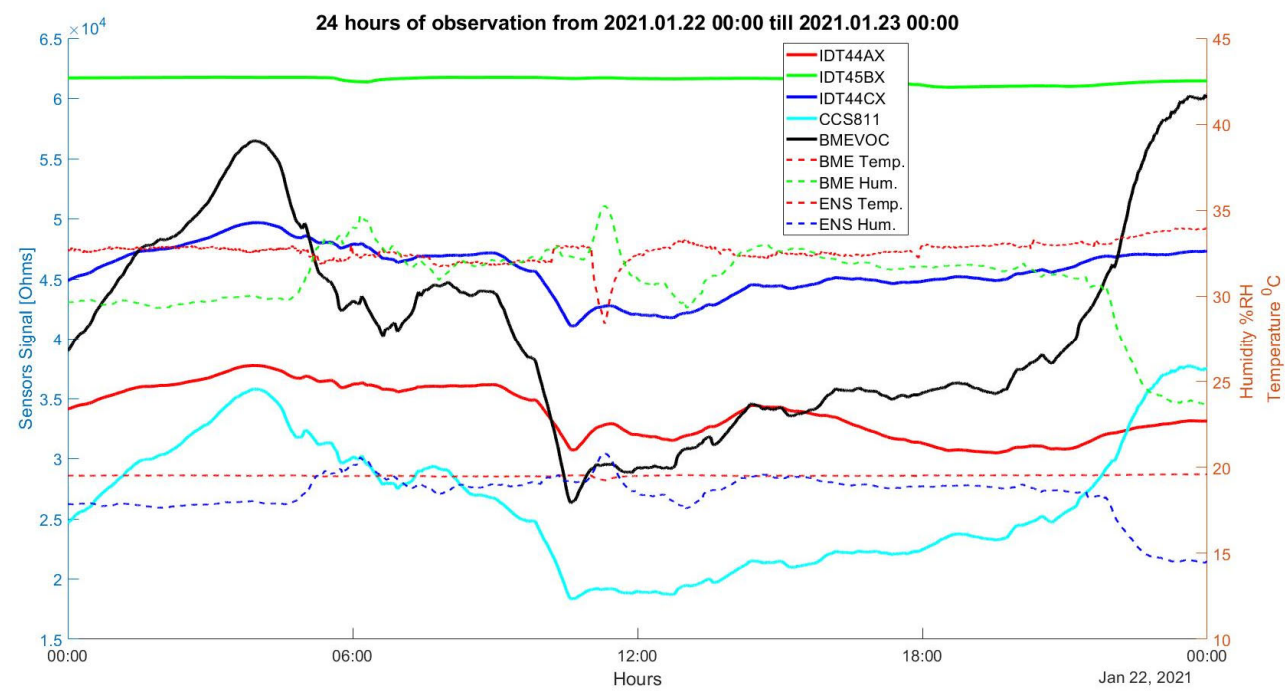

Figure 6. Environmental BME and ENS sensors, and gas sensor signals taken from a patient in the mechanical lung ventilation machine for $24 \mathrm{~h}$ of measurements.

\subsection{Data Exploration and Methodology}

In this section, we explore the data collected from eight patients using Principal Component Analysis (PCA) which allows for the visual inspection of the data structure on a new space of smaller dimension. In such a space, the orthogonal axes or Principal Components (PCs) are such that they capture sequentially decreasing amounts of data variance. Therefore, $\mathrm{PC} 1$ captures more variance than PC2, and so on. A PCA scores plot represents the data projected in the first two PCs of this PCA space (PC1 and PC2), which are thus the two PCs that capture the data variability the most. Indeed, PCA is a convenient way to represent multivariate data, as the PCs are linear combinations of the original sensors.

The dataset consists of sensor measurements from the breath of 10 patients across a few days. Data from two of the patients were discarded due to the short measurement time or bad data quality because of individual sensors' malfunction. Table 2 shows basic information about the patients. Notice the variability in the samples; the patient set includes both genders with ages within a 25-year range (51-76), BMI between 23 and 45, two clinical sites, intubated or not, two final conditions (deceased or survived), four different devices, and a variable number of days of measurements.

Table 2. Information about the patients.

\begin{tabular}{|c|c|c|c|c|c|c|c|c|c|}
\hline Patient Number & 2 & 3 & 4 & 5 & 6 & 7 & 8 & 9 & 10 \\
\hline Age & 67 & 71 & 61 & 64 & 55 & 54 & 54 & 76 & 51 \\
\hline Sex & $\mathrm{F}$ & $\mathrm{M}$ & $\mathrm{M}$ & $\mathrm{F}$ & $\mathrm{M}$ & $\mathrm{M}$ & $\mathrm{F}$ & $\mathrm{M}$ & $\mathrm{F}$ \\
\hline BMI & 35.2 & 24.7 & 23.1 & 23.5 & 30.86 & 30.9 & 37.2 & 38.7 & 44.6 \\
\hline $\begin{array}{l}\text { Noninvasive } \\
\text { Ventilation }\end{array}$ & No & No & Yes & Yes & Yes & Yes & Yes & Yes & Yes \\
\hline Intubation & Yes & Yes & Yes & No & No & Yes & Yes & No & No \\
\hline Deceased/Survived & $\mathrm{D}$ & $\mathrm{D}$ & $\mathrm{D}$ & S & S & $\mathrm{D}$ & $\mathrm{D}$ & S & $\mathrm{S}$ \\
\hline Clinical Site & $\mathrm{G}^{1}$ & $\mathrm{G}^{1}$ & $\mathrm{G}^{1}$ & $\mathrm{G}^{1}$ & $\mathrm{LIC}^{2}$ & $\mathrm{G}^{1}$ & $\mathrm{LIC}^{2}$ & $\mathrm{G}^{1}$ & $\mathrm{G}^{1}$ \\
\hline Start Date & $\begin{array}{c}9 \\
\text { December } \\
2020\end{array}$ & $\begin{array}{c}10 \\
\text { December } \\
2020\end{array}$ & $\begin{array}{c}17 \\
\text { December } \\
2020\end{array}$ & $\begin{array}{c}29 \\
\text { December } \\
2020\end{array}$ & $\begin{array}{l}11 \text { January } \\
2021\end{array}$ & $\begin{array}{l}9 \text { January } \\
2021\end{array}$ & $\begin{array}{c}22 \\
\text { February } \\
2021\end{array}$ & $\begin{array}{c}24 \\
\text { February } \\
2021\end{array}$ & $\begin{array}{l}26 \text { March } \\
2021\end{array}$ \\
\hline Stop Date & $\begin{array}{c}16 \\
\text { December } \\
2020\end{array}$ & $\begin{array}{c}16 \\
\text { December } \\
2020\end{array}$ & $\begin{array}{c}23 \\
\text { December } \\
2020\end{array}$ & $\begin{array}{c}31 \\
\text { December } \\
2020\end{array}$ & $\begin{array}{l}17 \text { January } \\
2021\end{array}$ & $\begin{array}{c}30 \text { January } \\
2021\end{array}$ & $\begin{array}{c}25 \\
\text { February } \\
2021\end{array}$ & $\begin{array}{c}27 \\
\text { February } \\
2021\end{array}$ & $\begin{array}{l}3 \text { April } \\
2021\end{array}$ \\
\hline Number of Days & 8 & 7 & 7 & 3 & 7 & 21 & 4 & 4 & 8 \\
\hline
\end{tabular}

${ }^{1}$ Gailezers Hospital; ${ }^{2}$ Latvia Infectology Centre.

Before the PCA computation, we performed a few pre-processing steps. First of all, we removed two of the IDT-ZMODA sensors due to an extremely high correlation among them. In addition, we removed the first three hours of measurements because the sensors 
were showing large transients due to not being warm enough for stable responses. Other performed tasks, such as reducing the correlation to environmental variables, are detailed in the Supplementary Material.

After the above-mentioned pre-processing steps, because we only had data from eight patients, we built a PCA model with the complete data set. Figure 7 shows the scores plot of a PCA model capturing 95\% of the variability of all patients' data from the first two days of measurements, resulting in four principal components (PCs), as shown in Figure 8. The loadings plot in Figure 9 shows the contribution of the sensors to the first two PCs. Unfortunately, we have quite different amounts of data for different patients, and for this reason, we chose to build the PCA model with the first two days of measurements of all the patients and visualize the evolution of a few patients (p6, p7 and p10) with respect to the model.
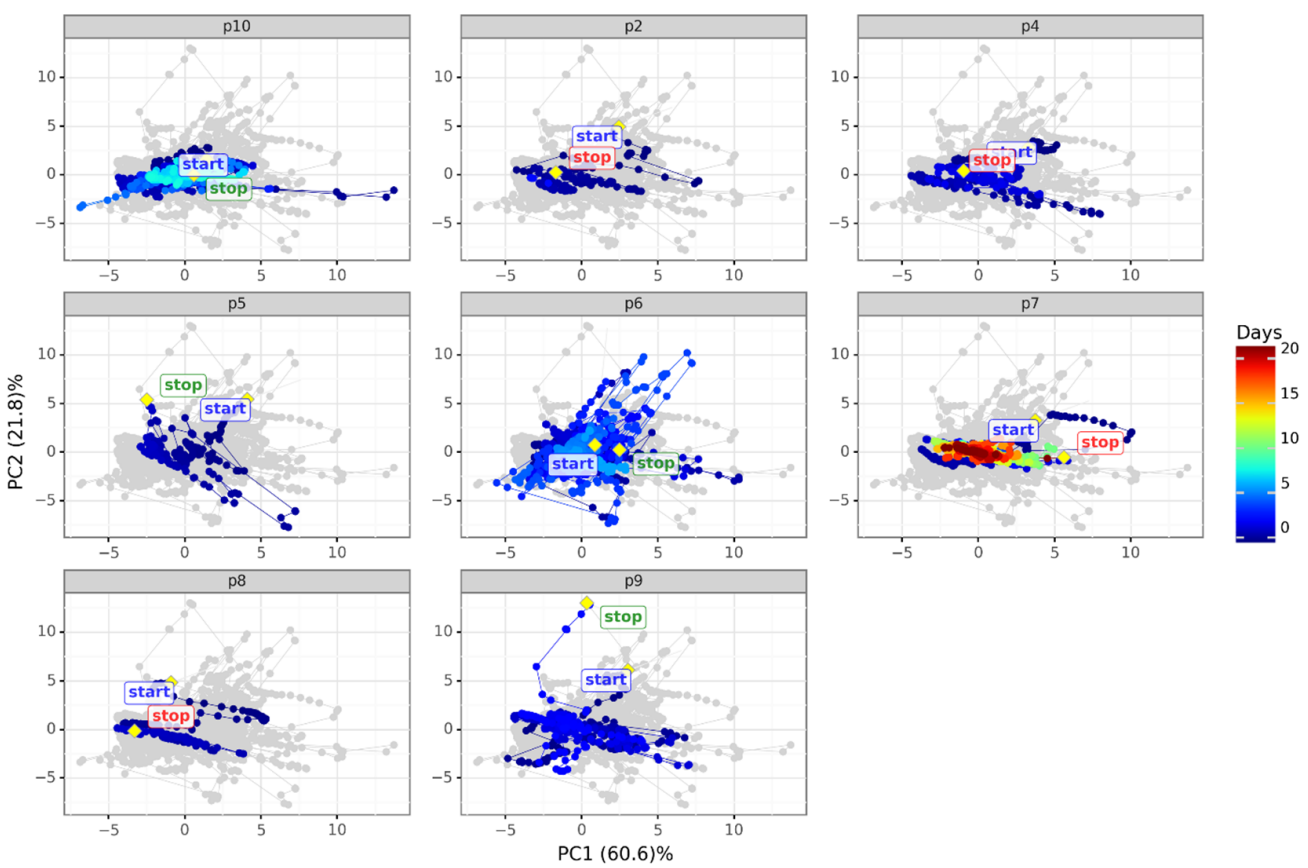

Figure 7. PCA scores plot per patient across time, capturing $95 \%$ of the variation of the patients' data in the first two days of measurements. The color of the STOP label indicates whether the patient survived (green) or died (red). The grey shadow indicates the silhouette of all the data in PC1 vs. PC2.

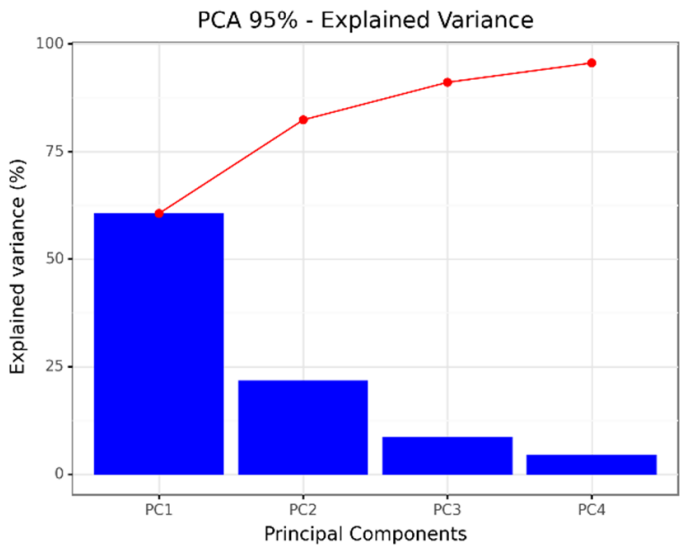

Figure 8. PCA explained variance plot. 


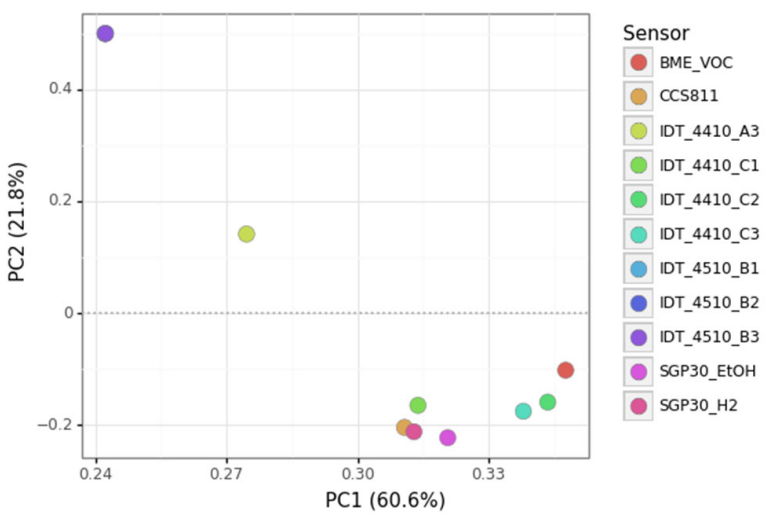

Figure 9. PCA loadings plot.

For better visualization of the PCA scores plot (Figure 7), the figure shows in grey the silhouette of the whole scores data, and then highlights the scores corresponding to each patient in separated panels with the patient number on top. The scores point colors correspond to the measurement time. Two large yellow points with a diamond shape and their corresponding label indicates the start (START) and the end (STOP) of the measurements. The color of the STOP label indicates the final status of the patient: survived (green) or deceased (red).

\section{Discussion}

A lot of effort is being made to get technological advances in developing novel analytical and sensing platforms for monitoring critically ill patients, including those with COVID-19. In this context, the scientific community believes that the exhaled breath analysis will realize its long-held potential becoming a revolutionary tool in personalized medicine and daily clinical practice [22,23]. Nano gas sensor-based approaches are effective in non-invasive diagnosis and the detection of the coronavirus because of their high detection capability, stability, reliability, design, and low cost [24,25]. The potential and different alternatives have been evaluated for the use of breath sensor technology in monitoring critically ill and COVID-19 patients. In fact, different types of nanomaterials have attracted the attention of the scientists in diagnosis, detection, monitoring, and therapy applications against COVID-19 [26]. Looking at the pathophysiology of COVID-19, there are several indicators that a coronavirus infection is detectable in the breath VOC pattern. The disease has been reported to cause a multitude of symptoms and can affect several organs. This supports the assumption that the metabolism is affected in multiple ways and that the volatile metabolite distribution is altered. Thus, a spectrum of VOCs could be used for monitoring the disease progression and effect of a therapeutic experimental drug, which would speed up the development of an efficient treatment for COVID-19.

We have designed and developed a novel breath analysis system based on gas sensor technology for monitoring severely ill COVID-19 patients in the mechanical lung ventilation machine. The sensor module is thought to enhance the monitoring features of the mechanical lung ventilation machine at hospital, providing environmental and gas sensors to analyze the exhaled breath of COVID-19 patients. The decision to connect the sensor system at the outlet of the ventilation equipment was approved based on the patient safety, efficacy of the sensor device, analysis of the breathing measurement data, and different considerations.

To test the performance of the new breath analysis system, we collected measurements from a set of eight patients ( 2 of the 10 patients discarded) during a variable period of time (from around 2 to 20 effective days). After few pre-processing steps to prepare the data (down-sampling, filtering, and decreasing the correlation with environmental variables), to explore the structure of the data we built a PCA model in the first two days of measurements for all the patients, retaining $95 \%$ of the variance in four features (Figure 8 ). 
The loadings plot (Figure 9) shows a high degree of correlation among sensors of similar type: IDT-ZMODB, SGP30, where some seem not to be present in the plot because the points are superimposed. Sensor redundancy is useful in sensor arrays for fault detection and correction, but this is not a priority in this application. In future system developments, we will consider alternative sensor technologies.

The PCA scores plot in Figure 7 shows the evolution of the condition of the patients in the first two PCs. Data for most patients correspond to the training set (dark blue for the first 2 days of measurements) and contain large variations that may correspond to changes in the patient status due to medicine intake, rotation in the patient's position, eating, or other episodes. We can see how the evolution of the patient's condition drives the 'stop' point far from the 'start' point (patients p9, p7, for example) or the contrary (p10, p6) in only two principal components. Data from several more patients are needed to find patterns that can help predict the patient's next status, such as worsening of his/her condition, so that the clinicians can act in advance. Such patterns would be based on breath analysis data, such as the one given by the system described here, or in combination with other parameters automatically collected from the patient.

From a technical point of view, many mechanical lung ventilators are available with different levels of complexity and sophistication. Because of the overwhelming health emergency caused due to COVID-19, the application of different rules in healthcare provision, including indications for artificial lung ventilation, is warranted [27]. This work reported on assessing the feasibility of implementing exhaled breath analysis through real-time sensor monitoring for critically ill COVID-19 patients in artificial ventilation equipment. The system followed the patient's state in real-time that could help to predict the risk of possible complications by studying the sensor signals. Therefore, it is possible to safely incorporate the developed breath sensor technology into the mechanical lung ventilation machine, and it has the potential to monitor critically ill patients, including those with COVID-19.

The urgency of the COVID-19 pandemic necessitates the use of such an innovative technology that can be used to forecast disease progression, to alert clinicians about the changes in patients' health, and to adjust their treatment. It is expected that, as the patient's sickness evolves, the VOC content in his/her breath changes. The study of the sensors signals monitoring the VOC pattern in the patient's breath during several days will allow to define and predict the patient's evolution in COVID-19, which in turn will help doctors to adjust therapy accordingly.

\section{Conclusions}

The preliminary results obtained are very promising and affirm that the developed breath sensor technology has potential in monitoring the course of severely ill patients, including COVID-19 patients, undergoing artificial ventilation. Furthermore, it is believed that the monitoring of the patients with severe COVID-19 infection with the sensor technology that is installed in the lung ventilation machine helps predict the course of the disease, and that it selects the subjects that are going to develop more severe complications. This will facilitate the decision-making on the management and treatment of severely ill COVID-19 patients. The sensor technology could be implemented for clinical use in the hospitals, therefore in the interests of the severely ill COVID-19 patients. This application may have wider implications beyond COVID-19 patients.

In summary, the initial clinical experiments and the data obtained so far in the patients with severe COVID-19 infection provide the proof and solution for the applicability of this sensor technology in these settings for long-term patient monitoring. In future work, the clinical data and other parameters will be registered and correlated to the sensor readings to evaluate the severity of the disease.

Supplementary Materials: The following supporting information can be downloaded at: https: / / www.mdpi.com/article/10.3390/diagnostics12020430/s1, File S1. 
Author Contributions: Conceptualization, M.P.B., M.P., O.Š., J.M. and M.L.; methodology, M.P.B., V.V., M.P., O.Š., A.S., I.K., N.I., D.B., J.M. and M.L.; software, M.P.B., V.V. and M.P.; formal analysis, M.P.B., V.V. and M.P.; investigation, V.V., I.S., O.Š., A.S., I.K., N.I., D.B., J.M. and M.L.; resources, M.P., O.Š., J.M. and M.L.; writing—original draft preparation, M.P.B. and M.L.; writing-review and editing, V.V., M.P., D.B., J.M. and M.L.; supervision, I.S., O.Š., J.M. and M.L.; project administration, I.S. and D.B.; funding acquisition, M.L. All authors have read and agreed to the published version of the manuscript.

Funding: This research was funded by the Ministry of Education and Science, Republic of Latvia, the Project No. VPP-COVID-2020/1-0023 “Clinical, biochemical, immunogenetic paradigms of COVID19 infection and their correlation with socio-demographic, etiological, pathogenetic, diagnostic, therapeutically and prognostically important factors to be included in guidelines". The sponsors had no role in study design, in collection or interpretation of data, in writing the report, or in the decision to submit the article for publication. The views expressed in this publication are those of the authors and not necessarily those of the Ministry. There was no commercial support for this study. All the authors vouch for the completeness and accuracy of the data presented and for the fidelity of the study to the protocol.

Institutional Review Board Statement: Ethical approval for this study was obtained from Central Medical Ethics committee of Latvia (Protocol No. 01-29.1/5034, approved on 1 September 2020 and Protocol No. for corrections 01-29.1.2/928, approved on 21 December 2020). All necessary patient/participant consent has been obtained and the appropriate institutional forms have been archived.

Informed Consent Statement: Informed consent was obtained from all subjects involved in the study.

Data Availability Statement: The data supporting the reported results are available on request from the corresponding authors.

Conflicts of Interest: The authors declare no conflict of interest.

\section{References}

1. Rackley, C.R. Monitoring During Mechanical Ventilation. Respir. Care 2020, 65, 832-846. [CrossRef]

2. Das, S.; Pal, M. Review-Non-Invasive Monitoring of Human Health by Exhaled Breath Analysis: A Comprehensive Review. J. Electrochem. Soc. 2020, 167, 037562. [CrossRef]

3. Cui, F.; Zhou, H.S. Diagnostic methods and potential portable biosensors for coronavirus disease 2019. Biosens. Bioelectron. 2020, 165, 112349. [CrossRef]

4. Sanchez, C.; Santos, J.P.; Lozano, J. Use of Electronic Noses for Diagnosis of Digestive and Respiratory Diseases through the Breath. Biosensors 2019, 9, 35. [CrossRef] [PubMed]

5. Wojnowski, W.; Dymerski, T.; Gebicki, J.; Namieśnik, J. Electronic Noses in Medical Diagnostics. Curr. Med. Chem. 2019, 26, 197-215. [CrossRef] [PubMed]

6. Haick, H.; Broza, Y.Y.; Mochalski, P.; Ruzsanyi, V.; Amann, A. Assessment, origin, and implementation of breath volatile cancer markers. Chem. Soc. Rev. 2014, 43, 1423-1449. [CrossRef] [PubMed]

7. Dixit, K.; Fardindoost, S.; Ravishankara, A.; Tasnim, N.; Hoorfar, M. Exhaled Breath Analysis for Diabetes Diagnosis and Monitoring: Relevance, Challenges and Possibilities. Biosensors 2021, 11, 476. [CrossRef]

8. Ray, B.; Parmar, S.; Vijayan, V.; Vishwakarma, S.; Datar, S. Detection of trace volatile organic compounds in spiked breath samples: A leap towards Breathomics. Nanotechnology 2022. [CrossRef]

9. Brock, B.; Kamysek, S.; Silz, J.; Trefz, P.; Schubert, J.K.; Miekisch, W. Monitoring of breath VOCs and electrical impedance tomography under pulmonary recruitment in mechanically ventilated patients. J. Breath Res. 2017, 11, 016005. [CrossRef]

10. Schnabel, R.; Fijten, R.; Smolinska, A.; Dallinga, J.; Boumans, M.L.; Stobberingh, E. Analysis of volatile organic compounds in exhaled breath to diagnose ventilator-associated pneumonia. Sci. Rep. 2015, 5, 17179. [CrossRef]

11. Schnabel, R.M.; Boumans, M.L.; Smolinska, A.; Stobberingh, E.E.; Kaufmann, R.; Roekaerts, P.M.H.J. Electronic nose analysis of exhaled breath to diagnose ventilator-associated pneumonia. Respir. Med. 2015, 109, 1454-1459. [CrossRef] [PubMed]

12. Grassin-Delyle, S.; Roquencourt, C.; Moine, P. Metabolomics of exhaled breath in critically ill COVID-19 patients: A pilot study. EBioMedicine 2021, 63, 103154. [CrossRef] [PubMed]

13. Takahama, A., Jr.; de Sousa, V.I.; Tanaka, E.E. Analysis of oral risk factors for ventilator-associated pneumonia in critically ill patients. Clin. Oral Investig. 2021, 25, 1217-1222. [CrossRef] [PubMed]

14. Maes, M.; Higginson, E.; Pereira-Dias, J.; Curran, M.D.; Parmar, S.; Khokhar, F. Ventilator-associated pneumonia in critically ill patients with COVID-19. Crit. Care 2021, 25, 25. [CrossRef]

15. Exline, M.C.; Stanacevic, M.; Bowman, A.S.; Gouma, P.I. Exhaled nitric oxide detection for diagnosis of COVID-19 in critically ill patients. PLoS ONE 2021, 16, e0257644. [CrossRef] 
16. Shan, B.; Broza, Y.Y.; Li, W.; Wang, Y.; Wu, S.; Liu, Z.; Wang, J.; Gui, S.; Wang, L.; Zhang, Z.; et al. Multiplexed Nanomaterial-Based Sensor Array for Detection of COVID-19 in Exhaled Breath. ACS Nano 2020, 14, 12125-12132. [CrossRef]

17. Konvalina, G.; Haick, H. Effect of humidity on nanoparticle-based chemiresistors: A comparison between synthetic and real-world samples. ACS Appl. Mater. Interfaces 2012, 4, 317-325. [CrossRef]

18. Blanchet, L.; Smolinska, A.; Baranska, A. Factors that influence the volatile organic compound content in human breath. J. Breath Res. 2017, 11, 016013. [CrossRef]

19. Jalal, A.H.; Alam, F.; Roychoudhury, S.; Umasankar, Y.; Pala, N.; Bhansali, S. Prospects and Challenges of Volatile Organic Compound Sensors in Human Healthcare. ACS Sens. 2018, 3, 1246-1263. [CrossRef] [PubMed]

20. Xiang, L.; Wu, S.; Hua, Q. Volatile Organic Compounds in Human Exhaled Breath to Diagnose Gastrointestinal Cancer: A Meta-Analysis. Front. Oncol. 2021, 11, 606915. [CrossRef]

21. Radogna, A.V.; Siciliano, P.A.; Sabina, S. A Low-Cost Breath Analyzer Module in Domiciliary Non-Invasive Mechanical Ventilation for Remote COPD Patient Monitoring. Sensors 2020, 20, 653. [CrossRef]

22. van de Kant, K.D.; van der Sande, L.J.; Jöbsis, Q.; van Schayck, O.C.; Dompeling, E. Clinical use of exhaled volatile organic compounds in pulmonary diseases: A systematic review. Respir. Res. 2012, 13, 117. [CrossRef] [PubMed]

23. Amor, R.E.; Nakhleh, M.K.; Barash, O. Breath analysis of cancer in the present and the future. Eur. Respir. Rev. $2019,28,190002$. [CrossRef] [PubMed]

24. Chintagunta, A.D.; Krishna, M.S.; Nalluru, S. Nanotechnology: An emerging approach to combat COVID-19. Emergent Mater. 2021, 4, 119-130. [CrossRef]

25. Adhikari, S.; Adhikari, U.; Mishra, A.; Guragain, B.S. Nanomaterials for diagnostic, treatment and prevention of COVID-19. Appl. Sci. Technol. Ann. 2020, 1, 155-164. [CrossRef]

26. Ghaemi, F.; Amiri, A.; Bajuri, M.Y.; Yuhana, N.Y.; Ferrara, M. Role of different types of nanomaterials against diagnosis, prevention and therapy of COVID-19. Sustain. Cities Soc. 2021, 72, 103046. [CrossRef]

27. Palacka, P.; Furka, S.; Furka, D.; Huzevka, T.; Gallik, D. Q-VENT-A Novel Device for Emergency Ventilation of the Lungs in Patients with Respiratory Failure due to Diseases such as COVID-19. Med. Devices Sens. 2020, 3, e10124. [CrossRef] 